\title{
Childhood trauma and bipolar spectrum: a population-based sample of young adults
}

\author{
Igor S. Vieira, ${ }^{1}$ (D) Fernanda Pedrotti Moreira, ${ }^{1}$ (D) Thaise C. Mondin, ${ }^{2}$ Taiane de A. Cardoso, ${ }^{3}$ \\ Karen Jansen, ${ }^{1}$ (D) Luciano D. de M. Souza, ${ }^{1}$ (D) Ricardo A. da Silva ${ }^{1}$ (D)
}

\begin{abstract}
Introduction: Childhood trauma has been suggested to be involved in susceptibility to bipolar disorder (BP). However, it remains unclear whether the occurrence of childhood trauma is differently distributed in subthreshold bipolar disorder (SBP).

Objective: To assess childhood trauma in young adults with SBP, as compared to young adults with BP and population controls (PC).

Method: This was a cross-sectional, population-based study. The Mini International Neuropsychiatric Interview (MINI) was used to define the groups with BP (subjects with a lifetime or current manic episode or lifetime or current hypomania with a history of a depressive episode), SBP (subjects with a history of hypomanic episode without lifetime or current depressive episode), and subjects without mood disorders (PC). Childhood trauma was assessed using de Childhood Trauma Questionnaire (CTQ). We investigated differences regarding childhood trauma across the three groups (BP, SBP and PC).

Result: Except for sexual abuse, all subtypes of childhood trauma remained associated with the BP group as compared to PC. Additionally, when we compared SBP and BP, significant differences were found only for emotional abuse. No significant differences were found in relation to childhood trauma between the SBP and PC groups after adjusting for confounding factors.

Conclusion: These findings suggest that investigating childhood trauma, with a particular focus on emotional abuse, could be considered a preventive measure and potentially improve the prognosis.

Keywords: Bipolar disorder, hypomania, childhood trauma, emotional abuse.
\end{abstract}

\section{Introduction}

Bipolar disorder (BP) is a heterogeneous illness involving episodes of severe mood disturbance that affects about $2 \%$ of the worldwide population. ${ }^{1} \mathrm{BP}$ is one of the leading causes of disability worldwide and causes substantial economic burden, thus being considered a public health problem. ${ }^{2,3}$ Also, it is associated with high rates of premature mortality due to suicide. ${ }^{4}$ However, epidemiological studies suggest that the estimates for this condition are conservative and neglect the growing evidence for a continuum of bipolar spectrum disorder. ${ }^{5}$ Patients who have pure hypomania without a prior major depressive episode are now classified as having "other specified bipolar and related disorder" in the Diagnostic and Statistical Manual of Mental Disorders, 5th edition (DSM-5), which is considered a subthreshold bipolar disorder (SBP), with a prevalence of $3.3 \% .^{6}$

Nevertheless, the experience of hypomanic symptoms does not necessarily indicate a future

\footnotetext{
${ }^{1}$ Programa de Pós-Graduação em Saúde e Comportamento, Universidade Católica de Pelotas, Pelotas, RS, Brazil. ${ }^{2}$ Pró-Reitoria de Assuntos Estudantis (PRAE), Universidade Federal de Pelotas, Pelotas, RS, Brazil. ${ }^{3}$ Department of Psychiatry and Behavioral Neurosciences, McMaster University, Hamilton, ON, Canada. Submitted Jun 05 2019, accepted for publication Nov 122019.

Suggested citation: Vieira IS, Pedrotti Moreira F, Mondin TC, Cardoso TA, Jansen K, Souza LDM, et al. Childhood trauma and bipolar spectrum: a populationbased sample of young adults. Trends Psychiatry Psychother. 2020;42(2):115-121. http://dx.doi.org/10.1590/2237-6089-2019-0046
} 
diagnosis of $\mathrm{BP}$, as in most cases these symptoms have a benign course, disappearing over time, without being associated with early mood disorders. ${ }^{5,7}$ Nevertheless, a subgroup of subjects with subthreshold expression of BP does make the transition to BP. ${ }^{5}$ There is evidence suggesting that an important factor that differentiates individuals who make the transition to BP from those who do not is the level of persistence of subthreshold expression of BP over time. ${ }^{5}$ Little is known about the factors that influence persistence of subthreshold expression of $\mathrm{BP}$, but it has been suggested that the onset and course of BP are affected by environmental interactions. ${ }^{8}$

Identification of the environmental determinants of $\mathrm{BP}$ is a major challenge, with implications for the identification of subjects at risk in an early stage of the development of the disorder, making early intervention possible. The occurrence of traumatic events during childhood is probably the most promising environmental determinant so far investigated, and it has been associated with negative outcomes in BP, e.g., early illness onset, worse lifetime course, higher number of comorbid mental disorders, suicide attempts, suicide risk, and treatment resistance. ${ }^{9-11}$ Despite the consistent finding of a relationship between childhood trauma and $\mathrm{BP}$, few studies have been conducted to assess the association between childhood stressors and SBP.

Thus, the aim of this study was to assess childhood trauma in young adults diagnosed with SBP as compared to young adults with BP and population controls (PC). Our main hypothesis was that individuals with BP developed full criteria for this disorder because they suffered more traumas in childhood as compared to individuals with SBP. A secondary hypothesis was that there would be few significant differences between the SBP and BP groups, suggesting that they are part of the same spectrum, presenting worse clinical characteristics and higher occurrence of childhood trauma when compared to PC.

\section{Method}

\section{Design and participants}

This was a cross-sectional study corresponding to the second wave of a population-based cohort study. The full description of the first wave can be found elsewhere. ${ }^{12}$ The inclusion criteria at baseline were: 1 ) to be between 18 and 24 years old; and 2) to live in the urban area of Pelotas in the period from 2007 to 2009. The only exclusion criterion was having a severe cognitive disability that would preclude understanding of the instruments. The second wave was undertaken between 2012 and 2014, at a mean of 5 years after the first one. All young adults that participated in the first phase ( $n=1,560$ ) were invited to return for a followup assessment; of the original participants, 1,244 individuals were located. Subjects were informed about the research objectives and signed a new informed consent form specifically designed for the second wave. Respondents who had a psychiatric disorder were referred for treatment at the mental health outpatient clinic of Universidade Católica de Pelotas (UCPel). This study was approved by the research ethics committee of UCPel (protocol 2008/118). Further details can be found in previously published reports. ${ }^{13}$

\section{Instruments}

Socioeconomic evaluation of the participants was performed through the classification from the Brazilian Association of Research Companies (Associação Brasileira de Empresas de Pesquisa - ABEP), ${ }^{14}$ which is based on the total number of material goods and the householder's educational level. In this classification, A refers to the highest socioeconomic class, and $E$ to the lowest one.

Tobacco and alcohol abuse/dependence were assessed using the Alcohol, Smoking and Substance Involvement Screening Test (ASSIST). ${ }^{15}$ This instrument consists of eight questions about the use of tobacco, alcohol, cannabis, cocaine, amphetaminetype stimulants, inhalants, sedatives, hallucinogens, opiates and "other drugs." The substance abuse/ dependence variable is calculated from the sum of the scores obtained for each substance class. A score of 0-3 is considered to be indicative of occasional use, and a score of 4 or higher is indicative of abuse/dependence. This instrument has been validated and adapted for the Brazilian population. ${ }^{15}$

Childhood trauma was evaluated using the Childhood Trauma Questionnaire (CTQ), in a version adapted to the Brazilian population by Grassi-Oliveira. ${ }^{16}$ The CTQ is a 28-item, self-report measure widely used to assess history of childhood abuse and neglect. This instrument can be administered to adolescents (12 years or older) and adults, investigating five traumatic components: emotional, sexual and physical abuse, and emotional and physical neglect. It uses a 5-item Likert scale. ${ }^{16}$

Current and lifetime psychiatric disorders were assessed using the Mini International Neuropsychiatric Interview 5.0 (MINI), ${ }^{17}$ administered by well-trained psychologists. We defined our groups as follows: subjects without mood disorders (PC); subjects with BP type I or BP type II (BP-I or BP-II); subjects with a history of hypomanic episode without lifetime or current 
depressive episode (SBP). This last group was defined based on the category described in DSM-5 as "other specified bipolar and related disorders," describing the third diagnostic criterion: "hypomanic episode, lasting for more than 4 days, without prior major depressive episode." Finally, for the proposal of this study, subjects with a diagnosis of depression without a history of manic or hypomanic episode were excluded from the analyses $(n=317)$.

\section{Statistical analysis}

Data were collected using Open Data Kit Collect 1.1.7 and then transferred to the Statistical Package for the Social Sciences (SPSS) version 25 for Windows, where the statistical analyses were conducted. Descriptive data were expressed as mean and standard deviation or absolute and relative frequency. The bivariate analysis was conducted using the chi-square test and the one-way analysis of variance (ANOVA), followed by Bonferroni's post-hoc correction. BP-I and BP-II were placed in the same category mainly because of the lack of power to investigate them separately. The multivariate analysis was conducted using Poisson regression to adjust the analysis for potential confounders. In this analysis, the diagnostic groups (PC vs. BP; SBP vs. BP; and PC vs. $\mathrm{SBP}$ ) were considered as dependent variables, and the CTQ subscales as independent variables. The trauma subtypes were included in the model one by one, considering as possible confounding factors any variable associated with the diagnostic groups and CTQ subscales with $\mathrm{p}<0.20$. In all statistical tests, associations were considered significant when $\mathrm{p}<0.05$.

\section{Results}

In total, 927 young adults were included in the study. Of these, $51.9 \%$ were women; the mean age of the total sample was $25.81 \pm 2.16$ years; $71.8 \%$ were Caucasian; $23.1 \%$ reported tobacco abuse/dependence; and $24.6 \%$ were identified as having alcohol abuse/ dependence. Regarding the groups, 90 (9.7\%) subjects were diagnosed with $\mathrm{BP}, 21$ (2.3\%) with SBP, and 816 (88.0\%) were assigned to the PC group.

The demographic and clinical characteristics of the sample are shown in Table 1. The BP group showed a higher prevalence of women ( $p<0.001)$, lower educational level ( $p<0.001)$, lower socioeconomic status ( $p<0.001)$, higher prevalence of family history of psychiatric disorders $(p<0.001)$, higher prevalence of alcohol abuse $(p<0.001)$, higher prevalence of tobacco abuse $(p<0.001)$, and higher prevalence of suicide risk ( $p<0.001$ ) as compared to PC. In turn, subjects with SBP showed no significant differences regarding demographic and clinical characteristics as compared to the PC group. Finally, when the SBP and BP groups were compared, we found a higher prevalence of women in BP as compared to SBP ( $p<0.001)$, a higher prevalence of family history of psychiatry disorders $(p=0.021)$, and a higher prevalence of suicide risk ( $p=0.048$ ) (Table 1 ).

Table 2 shows the means and Bonferroni post-hoc correction of childhood trauma events in each group. Subjects with BP scored higher than PC for all subtypes of childhood trauma ( $p<0.05)$. In addition, subjects with $\mathrm{BP}$ also showed a higher score of childhood trauma as compared to SBP, for all subtypes of trauma, except for emotional and physical neglect, where there were no differences. We found no significant differences between groups PC and SBP regarding the subtypes of childhood trauma, except for emotional neglect, in which the subjects with SBP showed a higher score as compared to PC $(p=0.025)$.

In the multivariate Poisson regression analyses, the diagnostic groups (PC vs. BP, SBP vs. BP and PC vs. $\mathrm{SBP}$ ) were considered as dependent variables, and the CTQ subscales as independent variables.

Except for sexual abuse $(p=0.061$; adjusted for sex, ethnicity, educational level, socioeconomic status, alcohol abuse, tobacco abuse, and suicide risk), all subtypes of childhood trauma remained associated with the BP group as compared to PC: emotional abuse ( $p<0.001$; adjusted for sex, ethnicity, socioeconomic status, family history of psychiatric disorder, alcohol abuse, tobacco abuse, and suicide risk), physical abuse ( $p<0.001$; adjusted for educational level, socioeconomic status, family history of psychiatric disorder, alcohol abuse, tobacco abuse, and suicide risk), emotional neglect ( $p<0.001$; adjusted for educational level, socioeconomic status, family history of psychiatric disorder, alcohol abuse, tobacco abuse, and suicide risk), physical neglect ( $p=0.002$; adjusted for ethnicity, educational level, socioeconomic status, family history of psychiatric disorder, tobacco abuse, and suicide risk), and total CTQ ( $p<0.001$; adjusted for sex, ethnicity, educational level, socioeconomic status, family history of psychiatric disorder, alcohol abuse, tobacco abuse, and suicide risk). Additionally, when we compared SBP and BP, we found significant differences only for emotional abuse ( $p=0.022$; adjusted for sex, family history of psychiatric disorder, and suicide risk) (Table 3 ). No significant differences were found for childhood trauma scores between the SBP and PC groups after adjusting for confounding factors (Table 3). 
Table 1 - Demographic and clinical characteristics of the sample according to group: population control (PC), subthreshold bipolar disorder (SBP) and bipolar disorder (BP)

\begin{tabular}{|c|c|c|c|c|c|c|c|c|}
\hline Variables & $\begin{array}{c}\text { PC } \\
\mathrm{n}=\mathbf{8 1 6}\end{array}$ & $\begin{array}{c}\text { SBP } \\
n=21\end{array}$ & $\begin{array}{c}\text { BP } \\
\mathbf{n}=90\end{array}$ & $x^{2}$ & $\begin{array}{c}\text { Global } \\
\text { p-value }\end{array}$ & $\frac{\text { PC vs. BP }}{\text { p-value }}$ & $\begin{array}{c}\text { PC vs. } \\
\text { SBP } \\
\text { p-value }\end{array}$ & $\begin{array}{c}\begin{array}{c}\text { SBP vs } \\
\text { BP }\end{array} \\
\text { p-value }\end{array}$ \\
\hline Age (year), mean $\pm \mathrm{SD}$ & $25.83 \pm 2.16$ & $25.33 \pm 2.15$ & $25.78 \pm 2.11$ & - & $0.857 *$ & - & - & - \\
\hline Sex & & & & 22.172 & $<0.001$ & $<0.001$ & 0.075 & $<0.001$ \\
\hline Male & 407 (49.9) & $15(71.4)$ & $24(26.7)$ & & & & & \\
\hline Female & $409(50.1)$ & $6(28.6)$ & $66(73.3)$ & & & & & \\
\hline Ethnicity & & & & 3.589 & 0.166 & 0.058 & 1.000 & 0.614 \\
\hline Caucasian & $594(72.8)$ & $15(71.4)$ & $57(63.3)$ & & & & & \\
\hline Non-Caucasian & $222(27.2)$ & $6(28.6)$ & $33(36.7)$ & & & & & \\
\hline Educational level & & & & 25.489 & $<0.001$ & $<0.001$ & 0.069 & 0.492 \\
\hline Primary & $230(28.2)$ & $12(57.1)$ & $44(48.9)$ & & & & & \\
\hline Middle school & $232(28.4)$ & $3(14.3)$ & $24(26.7)$ & & & & & \\
\hline High school or over & $354(43.4)$ & $6(28.6)$ & $22(24.4)$ & & & & & \\
\hline Economic classification (ABEP) & & & & 29.478 & $<0.001$ & $<0.001$ & 0.250 & 0.360 \\
\hline$A+B$ (upper) & $476(58.5)$ & $9(42.9)$ & $34(38.2)$ & & & & & \\
\hline C & $323(39.7)$ & $12(57.1)$ & $47(52.8)$ & & & & & \\
\hline$D+E$ (lower) & $14(1.7)$ & $0(0.0)$ & $8(9.0)$ & & & & & \\
\hline Family psychiatric history & $380(47.7)$ & $9(42.4)$ & $62(70.0)$ & 16.284 & $<0.001$ & $<0.001$ & 0.682 & 0.021 \\
\hline Alcohol abuse & $187(23.0)$ & $6(28.6)$ & $35(39.3)$ & 11.718 & $<0.001$ & $<0.001$ & 0.600 & 0.455 \\
\hline Tobacco abuse & $161(19.8)$ & $7(33.3)$ & $46(51.7)$ & 50.958 & $<0.001$ & $<0.001$ & 0.173 & 0.128 \\
\hline Suicide risk & $20(2.5)$ & $2(9.5)$ & $37(41.1)$ & 203.651 & $<0.001$ & $<0.001$ & 0.102 & 0.048 \\
\hline
\end{tabular}

$\mathrm{ABEP}=$ Brazilian Association of Research Companies (Associação Brasileira de Empresas de Pesquisa) ${ }^{14}$; SD $=$ standard deviation. Data presented as $\mathrm{n}(\%)$ and analyzed using the chi-square test, unless otherwise specified.

* One-way analysis of variance (ANOVA).

Table 2 - Childhood trauma among population control (PC), subthreshold bipolar disorder (SBP) and bipolar disorder (BP)

\begin{tabular}{|c|c|c|c|c|c|c|c|}
\hline & \multirow{2}{*}{$\begin{array}{c}\text { PC } \\
\text { Mean (SD) }\end{array}$} & \multirow{2}{*}{$\begin{array}{c}\text { SBP } \\
\text { Mean (SD) }\end{array}$} & \multirow{2}{*}{$\begin{array}{c}\text { BP } \\
\text { Mean (SD) }\end{array}$} & \multicolumn{4}{|c|}{ Statistics } \\
\hline & & & & $\mathbf{F}$ & df & p-values & Direction \\
\hline CTQ emotional abuse & $6.77(2.52)$ & $7.09(1.72)$ & $10.08(4.32)$ & 59.046 & 2 & $<0.001$ & $\mathrm{BP}>\mathrm{PC}, * \mathrm{BP}>\mathrm{SBP} *$ \\
\hline СTQ physical abuse & $5.99(1.58)$ & $6.38(1.65)$ & $7.78(3.71)$ & 35.929 & 2 & $<0.001$ & $\mathrm{BP}>\mathrm{PC}^{*} * \mathrm{BP}>\mathrm{SBP}^{+}$ \\
\hline CTQ sexual abuse & $5.24(1.18)$ & $5.19(0.67)$ & $6.24(2.79)$ & 20.222 & 2 & $<0.001$ & $\mathrm{BP}>\mathrm{PC}_{1}^{+} \mathrm{BP}>\mathrm{SBP}^{\neq}$ \\
\hline СTQ emotional neglect & $7.61(3.13)$ & $9.00(3.93)$ & $10.55(4.42)$ & 32.822 & 2 & $<0.001$ & $\mathrm{BP}>\mathrm{PC}^{*} * \mathrm{SBP}>\mathrm{PC}^{\ddagger}$ \\
\hline CTQ physical neglect & $6.06(1.85)$ & $6.47(3.09)$ & $7.49(2.79)$ & 20.724 & 2 & $<0.001$ & $\mathrm{BP}>\mathrm{PC} *$ \\
\hline Total CTQ score & $38.29(8.54)$ & $41.33(10.19)$ & $49.75(13.34)$ & 59.385 & 2 & $<0.001$ & $\mathrm{BP}>\mathrm{PC}, * \mathrm{BP}>\mathrm{SBP} *$ \\
\hline
\end{tabular}

$\mathrm{CTQ}=$ Childhood Trauma Questionnaire; $\mathrm{df}=$ degrees of freedom; $\mathrm{F}=$ analysis of variance; $\mathrm{SD}=$ standard deviation.

${ }^{*} \mathrm{p}<0.001 ;{ }^{+} \mathrm{p}<0.05 ;{ }^{*} \mathrm{p}<0.01$.

Table 3 - Multivariate analysis using Poisson regression to assess the association between childhood trauma and the groups: population control (PC), subthreshold bipolar disorder (SBP) and bipolar disorder (BP)

\begin{tabular}{|c|c|c|c|c|c|c|c|c|c|}
\hline \multirow{2}{*}{ CTQ } & \multicolumn{3}{|c|}{ PC* $\times$ BP } & \multicolumn{3}{|c|}{ SBP* $\times$ BP } & \multicolumn{3}{|c|}{ PC* $\times$ SBP } \\
\hline & PR & $95 \% \mathrm{CI}$ & $\mathbf{p}$ & PR & $95 \% \mathrm{CI}$ & $\mathbf{p}$ & PR & $95 \% \mathrm{CI}$ & $\mathbf{p}$ \\
\hline CTQ emotional abuse & 1.11 & $(1.07-1.15)$ & $<0.001$ & 1.02 & $(1.00-1.03)$ & 0.039 & 1.03 & $(0.94-1.13)$ & 0.435 \\
\hline СTQ physical abuse & 1.08 & $(1.04-1.14)$ & 0.001 & 1.00 & $(0.99-1.02)$ & 0.289 & 1.07 & $(0.93-1.24)$ & 0.309 \\
\hline CTQ sexual abuse & 1.05 & $(0.99-1.12)$ & 0.061 & 1.01 & $(0.99-1.02)$ & 0.088 & 0.96 & $(0.69-1.33)$ & 0.814 \\
\hline CTQ emotional neglect & 1.09 & $(1.04-1.14)$ & $<0.001$ & 1.00 & $(0.99-1.02)$ & 0.338 & 1.07 & $(0.96-1.19)$ & 0.177 \\
\hline CTQ physical neglect & 1.09 & $(1.03-1.17)$ & 0.002 & 1.01 & $(0.97-1.04)$ & 0.462 & 1.02 & $(0.80-1.29)$ & 0.866 \\
\hline Total CTQ score & 1.02 & $(1.01-1.04)$ & $<0.001$ & 1.00 & $(0.99-1.00)$ & 0.198 & 1.01 & $(0.98-1.05)$ & 0.285 \\
\hline
\end{tabular}

PR = prevalence ratio; $95 \% \mathrm{CI}=$ confidence interval; CTQ = Childhood Trauma Questionnaire.

$*$ Reference category. 


\section{Discussion}

In our sample, subjects with SBP presented sociodemographic and clinical characteristics similar to those of the PC group. Regarding trauma, there were also no differences between these two groups (SBP and PC). However, subjects with BP scored higher in all CTQ subscales compared to PC, and higher on emotional abuse compared to SBP.

Although pure hypomania is classified as a SBP subcategory in DSM-5, our results showed that the SBP group was similar to the PC group, contrary to our initial hypothesis. A previous study examining risk factors associated with subliminal BP showed that this manifestation of BP presented worse behavioral outcomes (substance abuse, suicide risk, and others) compared to PC. ${ }^{18}$ These contradictory results could be explained by the broader definition of subliminal BP adopted in that study, namely, including dysthymia, minor depression or recurrent brief depression associated with hypomania, whereas our classification included only hypomania, without current or lifetime depressive episode. ${ }^{6,12}$

Our study is in line with the findings of previous studies in that subjects with a diagnosis of BP reported higher rates of childhood trauma than PC. ${ }^{10,19-21}$ It is consensus in the literature that traumatic experiences are associated with the development and a worse course of BP. 9,10 In this sense, a recent meta-analysis showed that patients with BP with a history of childhood maltreatment presented greater mania severity, greater depression severity, greater psychosis severity, higher risk of comorbidity with post-traumatic stress disorder, anxiety disorders and substance misuse disorders, earlier age of BP onset, higher risk of rapid cycling, greater number of manic episodes, greater number of depressive episodes, and higher risk of suicide attempt, as compared to patients with BP without childhood maltreatment. ${ }^{9}$ These data reinforce the importance of assessing childhood trauma in individuals with BP, because it can potentially assist clinicians in their treatment strategies and improve the care of patients with BP.

Exploring the subtypes of trauma, we did not find significant differences in the sexual abuse subtype between the PC and BP groups, which is similar to previous reports and also supported by a study that found sexual abuse to be the least reported form of abuse by patients with BP. ${ }^{19,22,23}$ Moreover, authors have demonstrated that BP-I patients differ significantly from healthy controls for sexual abuse, and BP-II patients differ from healthy controls for emotional neglect. ${ }^{24}$ However, in our study, we did not explore differences regarding trauma experience between BP-I and BP-II. Even though we did not find differences for sexual abuse, our results showed that the mean of all the other types of childhood trauma were higher in BP as compared to the PC group, especially emotional abuse. In agreement with these findings, Garno et al. ${ }^{25}$ identified histories of childhood abuse in $51 \%$ of a cohort of 100 adults with $\mathrm{BP}$, and emotional abuse was the most frequent type of trauma. A retrospective study has also found that emotional abuse was the subtype of trauma that had the highest effect in patients with BP compared to controls. ${ }^{26}$

Interestingly, a study including 4,547 young adults aiming to develop and validate criteria for the definition of milder expressions of BP-II and hypomania showed some similarities with our findings. ${ }^{6}$ The prevalence of pure hypomania (no history of depression) was similar to ours: they found $3.3 \%,{ }^{6}$ vs. $2.3 \%$ in our sample. Additionally, that study also showed that individuals with pure hypomania were similar to individuals without mood disorders regarding family history of mania or depression, tobacco abuse, and suicide risk $^{6}$ - again in line with our findings. This suggests that pure hypomania could be a less severe condition due to the similarity of its characteristics with those of individuals without mood disorders.

Despite the consensus in the literature regarding the impact of trauma on $\mathrm{BP}$, this relationship is still unknown in SBP. Our study showed that trauma was not associated with SBP when compared to PC, disagreeing with a previous study. ${ }^{18}$ However, comparisons with previous investigations are difficult to make, because of the wide range of possible SBP classifications and definitions employed. However, we hypothesized that this non-significant association was due to the sociodemographic and clinical similarity of the two groups.

Conversely, trauma seems to have some influence on BP when compared with the SBP group. Among all subtypes of childhood trauma assessed, only emotional abuse was a significant predictor of BP compared to SBP after adjusting the analysis for confounding factors. In this sense, it is possible that exposure to emotional abuse could contribute to the development of fullblown criteria for BP. This result can be explained by the fact that childhood trauma is linked to the persistence of subthreshold symptoms of BP, which in turn could contribute to the development of the disease. ${ }^{18,27}$ Taken together, these results suggest that emotional abuse specifically may influence the expression of symptoms in BP most seriously. Moreover, they show that childhood trauma subtypes can have different impacts at different stages of BP development, corroborating a meta-analysis 
that suggests that childhood trauma is associated with particular forms of the bipolar symptomatology. ${ }^{28}$

Our findings should be interpreted considering some limitations. First, data about childhood trauma were collected retrospectively. It should nevertheless be noted that despite the possibility of memory bias, the CTQ is a widely used and statistically robust questionnaire. Second, the diagnosis of SBP was limited to pure hypomania, which hinders comparisons between our data and those of previous studies. Finally, we had a small sample size in the SBP group, and we did not assess some clinical features associated with the course of BP, such as BP subtype, number of episodes, predominant polarity and duration of illness. Thus, the hypothesis about the course of SBP needs further investigation in a longitudinal study, including a large and robust sample. Despite these limitations, the main strengths of this study are its population-based study design, including a sample in an age range in which $\mathrm{BP}$ has a probability of having recently manifested. In addition, to the best of our knowledge, this was the first study to investigate childhood trauma in a populationbased study differentiating between BP and SBP.

Our main finding was that the presence of emotional abuse was the only subtype of trauma that significantly differentiated BP from SBP, which partially confirms our hypothesis that individuals with BP suffered more trauma, and then developed full criteria for the diagnosis. These findings suggest that childhood trauma deserves more attention in psychiatric clinical practice and scientific research. It would be interesting to include systematic assessment of childhood trauma in high-risk populations (e.g., patients with SBP, bipolar patients' offspring) in clinical practice, with a particular focus on emotional abuse, as a preventive measure. Longitudinal studies are necessary not only to identify the actual effects of childhood trauma on the lives of young adults, but also to better understand the consequences that those traumatic experiences may bring to the course of BP.

\section{Acknowledgements}

This study was partly funded by Fundação de Amparo à Pesquisa do Estado do Rio Grande do Sul (FAPERGS) and Conselho Nacional de Desenvolvimento Científico e Tecnológico (CNPq).

\section{Disclosure}

No conflicts of interest declared concerning the publication of this article.

\section{References}

1. Radua J, Grunze H, Amann BL. Meta-analysis of the risk of subsequent mood episodes in bipolar disorder. Psychother Psychosom. 2017;86:90-8.

2. Krahn GL. WHO World Report on Disability: a review. Disabil Health J. 2011;4:141-2.

3. Matza L, De Lissovoy G, Sasané R, Pesa J, Mauskopf J. The impact of bipolar disorder on work loss. Drug Benefit Trends. 2004; 16:476-81.

4. Hayes J, Miles J, Walters K, King M, Osborn D. A systematic review and meta $\square$ analysis of premature mortality in bipolar affective disorder. Acta Psychiatr Scand. 2015;131:417-25.

5. Merikangas KR, Jin R, He JP, Kessler RC, Lee S, Sampson NA, et al. Prevalence and correlates of bipolar spectrum disorder in the world mental health survey initiative. Arch Gen Psychiatry. 2011;68:241-51.

6. Angst J, Gamma A, Benazzi F, Ajdacic V, Eich D, Rössler W. Toward a re-definition of subthreshold bipolarity: epidemiology and proposed criteria for bipolar-II, minor bipolar disorders and hypomania. J Affect Disord. 2003;73:133-46.

7. Regeer E, Krabbendam L, De Graaf R, Ten Have M, Nolen W, Van Os J. A prospective study of the transition rates of subthreshold (hypo) mania and depression in the general population. Psychol Med. 2006;36:619-27.

8. Post RM, Leverich GS, Xing G, Weiss SR. Developmental vulnerabilities to the onset and course of bipolar disorder. Dev Psychopathol. 2001;13:581-98.

9. Agnew-Blais J, Danese A. Childhood maltreatment and unfavourable clinical outcomes in bipolar disorder: a systematic review and meta-analysis. Lancet Psychiatry. 2016;3:342-9.

10. Bortolato $B$, Köhler CA, Evangelou E, León $\square$ Caballero J, Solmi M, Stubbs B, et al. Systematic assessment of environmental risk factors for bipolar disorder: an umbrella review of systematic reviews and meta $\square$ analyses. Bipolar Disord. 2017;19:84-96.

11. Etain B, Henry C, Bellivier F, Mathieu F, Leboyer M. Beyond genetics: childhood affective trauma in bipolar disorder. Bipolar Disord. 2008;10:867-76.

12. Jansen $K$, da Costa Ores L, de Azevedo Cardoso T, da Costa Lima R, de Mattos Souza LD, da Silva Magalhães PV, et al. Prevalence of episodes of mania and hypomania and associated comorbidities among young adults. J Affect Disord. 2011;130:328-33.

13. Vieira D, de Azevedo Cardoso $T$, Mondin $T$, Jansen $K$, da Silva R, de Mattos Souza $L$, et al. Mood disorders and prospective suicidality in young adults: a population $\square$ based cohort study. Acta Psychiatr Scand. 2018;137:109-15.

14. Associação Brasileira de Empresas de Pesquisa. Critério de Classificação Econômica. São Paulo: ABEP; 2008.

15. Henrique IFS, De Micheli D, Lacerda RBd, Lacerda LAd, Formigoni MLOdS. Validation of the Brazilian version of alcohol, smoking and substance involvement screening test (ASSIST). Rev Assoc Med Bras. 2004;50:199-206.

16. Grassi-Oliveira R, Stein LM, Pezzi JC. [Translation and content validation of the Childhood Trauma Questionnaire]. Rev Saude Publica. 2006;40:249-55.

17. Amorim P. Mini International Neuropsychiatric Interview (MINI): validação de entrevista breve para diagnóstico de transtornos mentais. Braz J Psychiatry. 2000;22:106-15.

18. Grandin LD, Alloy LB, Abramson LY. Childhood stressful life events and bipolar spectrum disorders. J Soc Clin Psychol. 2007;26:46078.

19. Etain B, Mathieu F, Henry C, Raust A, Roy I, Germain A, et al. Preferential association between childhood emotional abuse and bipolar disorder. J Trauma Stress. 2010;23:376-83.

20. Fowke A, Ross $S$, Ashcroft $K$. Childhood maltreatment and internalized shame in adults with a diagnosis of bipolar disorder. Clin Psychol Psychother. 2012;19:450-7.

21. Etain B, Lajnef $M$, Henrion A, Dargél A, Stertz L, Kapczinsk $\mathrm{F}$, et al. Interaction between SLC6A4 promoter variants and childhood trauma on the age at onset of bipolar disorders. Sci Rep. 2015;5:16301.

22. Watson S, Gallagher P, Dougall D, Porter R, Moncrieff J, Ferrier IN et al. Childhood trauma in bipolar disorder. Aust N Z J Psychiatry. 2014;48:564-70.

23. Larsson S, Andreassen OA, Aas M, Røssberg JI, Mork E, Steen $\mathrm{NE}$, et al. High prevalence of childhood trauma in patients with schizophrenia spectrum and affective disorder. Compr Psychiatry. 2013;54:123-7. 
24. Janiri D, Sani G, Danese E, Simonetti A, Ambrosi E, Angeletti G, et al. Childhood traumatic experiences of patients with bipolar disorder type I and type II. J Affect Disord. 2015;175:92-7.

25. Garno JL, Goldberg JF, Ramirez PM, Ritzler BA. Impact of childhood abuse on the clinical course of bipolar disorder. $\mathrm{Br}$ J Psychiatry. 2005; 186:121-5.

26. Leboyer M, Etain B, Mathieu F, Henry C, Jamain S, Bellivier F. Childhood affective trauma in bipolar affective disorder. Bipolar Disord. 2007:9:9-10.

27. Tijssen MJ, Van Os J, Wittchen $\mathrm{H}-\mathrm{U}$, Lieb R, Beesdo K, Wichers $M$. Risk factors predicting onset and persistence of subthreshold expression of bipolar psychopathology among youth from the community. Acta Psychiatr Scand. 2010;122:255-66.
28. Palmier-Claus J, Berry K, Bucci S, Mansell W, Varese F. Relationship between childhood adversity and bipolar affective disorder: systematic review and meta-analysis. Br J Psychiatry. 2016;209:454-9.

\section{Correspondence:}

Ricardo A. da Silva

Rua Gonçalves Chaves, 373, sala 424C, Centro

96015-560 - Pelotas, RS - Brazil

Tel.: +55 5321188404

E-mail: ricardo.as@uol.com.br 